\title{
Competitive Integration of Visual and Goal-related Signals on Neuronal Accumulation Rate: A Correlate of Oculomotor Capture in the Superior Colliculus
}

\author{
Brian J. White ${ }^{1}$, Robert A. Marino ${ }^{1}$, Susan E. Boehnke ${ }^{1}$, Laurent Itti $^{2}$, \\ Jan Theeuwes ${ }^{3}$, and Douglas P. Munoz ${ }^{1}$
}

\begin{abstract}
The mechanisms that underlie the integration of visual and goal-related signals for the production of saccades remain poorly understood. Here, we examined how spatial proximity of competing stimuli shapes goal-directed responses in the superior colliculus (SC), a midbrain structure closely associated with the control of visual attention and eye movements. Monkeys were trained to perform an oculomotor-capture task [Theeuwes, J., Kramer, A. F., Hahn, S., Irwin, D. E., \& Zelinsky, G. J. Influence of attentional capture on oculomotor control. Journal of Experimental Psychology. Human Perception and Performance, 25, 1595-1608, 1999], in which a target singleton was revealed via an isoluminant color change in all but one item. On a portion of the trials, an additional salient item abruptly appeared near or far from the target. We quantified how spatial proximity between the abrupt-onset and the target shaped the goal-directed response. We found that the appearance of an abrupt-onset near
\end{abstract}

\section{INTRODUCTION}

Most of us take for granted that our eyes are always moving in response to external stimuli and internal goals. Accordingly, visual attention can be voluntarily directed (i.e., goal-directed) but it is often involuntarily "captured" by goal-irrelevant stimuli during critical day-to-day actions (Leonard \& Luck, 2011; Ludwig, Ranson, \& Gilchrist, 2008; de Fockert, Rees, Frith, \& Lavie, 2004; Theeuwes, De Vries, \& Godijn, 2003). In this study, we examined how competing visual and goal-related neuronal signals interact to influence oculomotor behavior during target selection.

There is evidence supported by several biologically inspired models that the superior colliculus (SC) plays an important role in resolving competitive interactions between visual and goal-related processes (Marino, Trappenberg, Dorris, \& Munoz, 2012; Bompas \& Sumner, 2011; Meeter, Van der Stigchel, \& Theeuwes, 2010; Dorris, Olivier, \& Munoz, 2007; Godijn \& Theeuwes,

\footnotetext{
${ }^{1}$ Queen's University, Kingston, Ontario, Canada, ${ }^{2}$ University of Southern California, Los Angeles, ${ }^{3}$ Vrije Universiteit, Amsterdam, The Netherlands
}

the target induced a transient decrease in goal-directed discharge of SC visuomotor neurons. Although this was indicative of spatial competition, it was immediately followed by a rebound in presaccadic activation, which facilitated the saccadic response (i.e., it induced shorter saccadic RT). A similar suppression also occurred at most nontarget locations even in the absence of the abrupt-onset. This is indicative of a mechanism that enabled monkeys to quickly discount stimuli that shared the common nontarget feature. These results reveal a pattern of excitation/inhibition across the SC visuomotor map that acted to facilitate optimal behavior - the short duration suppression minimized the probability of capture by salient distractors, whereas a subsequent boost in accumulation rate ensured a fast goal-directed response. Such nonlinear dynamics should be incorporated into future biologically plausible models of saccade behavior.

2002; Trappenberg, Dorris, Munoz, \& Klein, 2001). Neurons in the superficial SC layers (SCs) receive input predominantly from the retina and visual cortex, whereas neurons in the intermediate SC layers (SCi) integrate multisensory, cognitive, and motor information from several cortical and subcortical brain areas (see White \& Munoz, 2011b, for a recent review). The SCi in turn projects directly to the brainstem saccade generator (Rodgers, Munoz, Scott, \& Paré, 2006; Sparks, 2002). In the SCs and $\mathrm{SCi}$, visual onsets are represented by a transient burst of action potentials beginning about $50 \mathrm{msec}$ from the onset of a stimulus in a neuron's response field (RF). This transient response is associated with a momentary facilitation of spatial attention at the stimulus location (Fecteau \& Munoz, 2005). In the SCi, goal-related signals are represented by sustained low frequency activation, which is associated with cognitive processes such as movement preparation (Li \& Basso, 2008; Dorris \& Munoz, 1998; Munoz \& Wurtz, 1995; Glimcher \& Sparks, 1992) and covert spatial attention (Lovejoy \& Krauzlis, 2010; Ignashchenkova, Dicke, Haarmeier, \& Thier, 2004; Kustov \& Robinson, 1996). Importantly, there is strong evidence that the SCi is directly involved in the target selection 
process (White \& Munoz, 2011a; Port \& Wurtz, 2009; Kim \& Basso, 2008; Shen \& Paré, 2007; Krauzlis, Liston, \& Carello, 2004; McPeek \& Keller, 2004) and the competitive interaction associated with suppressing undesired visual signals from interrupting saccade goals (White, Theeuwes, \& Munoz, 2012; Dorris et al., 2007).

Conceptual models of saccade initiation have long postulated the idea of an oculomotor "decision signal" that accumulates linearly from some baseline toward a threshold (Carpenter, 1988). Under this framework, differences in saccadic RT (SRT) are attributable to differences in either baseline activation, accumulation rate (rate of rise), or threshold. Neurophysiological support for this has been found in the FEFs (Purcell, Schall, Logan, \& Palmeri, 2012; Hanes \& Schall, 1996) and the SC (Paré \& Hanes, 2003; see also Basso \& Wurtz, 1997). Computational models of the SCi have extended this idea by examining the spatial interaction between visual and goal-related signals within a dynamic competitive framework (Marino, Trappenberg, et al., 2012; Bompas \& Sumner, 2011; Meeter et al., 2010; Godijn \& Theeuwes, 2002; Trappenberg et al., 2001). An assumption underlying these models is that visual and goal-related signals mutually inhibit or excite one another depending on the spatial proximity of their corresponding population responses or "point images" (Marino, Rodgers, Levy, \& Munoz, 2008; McIlwain, 1986); that is, the local population of neurons activated by a given stimulus (see Methods). Spatially overlapping point images are believed to be mutually excitatory, thereby facilitating saccade initiation. Spatially nonoverlapping point images are believed to be mutually inhibitory, thereby delaying saccade initiation. Computational models that employ this type of spatial interaction have successfully accounted for many saccadic behaviors, in particular the variation in SRT associated with multiple competing stimuli (Marino, Trappenberg, et al., 2012). The primary mechanism proposed to account for this interaction is a lateral neural network believed to exist in the SCi (Isa et al., 2009; Dorris et al., 2007; Meredith \& Ramoa, 1998; Munoz \& Istvan, 1998; Behan \& Kime, 1996). However, it is not entirely known how this spatial interaction shapes the accumulation of goal-directed activity in the SCi. Previous models have postulated a linear accumulation of neuronal activity toward a threshold for saccade initiation (Carpenter, 1988), but the limitations of a linear mechanism are evident when one observes actual neuronal discharge patterns under various conditions and task constraints.

In this study, we examined how the spatial proximity between competing signals in the SCi shapes the goaldirected response, using a task designed to dissociate visual from goal-related activation-the oculomotor-capture task (Theeuwes, Kramer, Hahn, Irwin, \& Zelinsky, 1999; Figure 1). In this task, the observer fixates a central stimulus followed by the appearance of an array of homogeneous peripheral "placeholder" stimuli, each representing a potential target location. After a delay, a target singleton is revealed via an isoluminant color change in all but one of the place- holders. Simultaneously, on a portion of the trials, an additional salient item (the same color as the distractors) appears abruptly either near (Figure 1B; local abrupt-onset) or far (Figure 1C; remote abrupt-onset) from the goal. The observer is required to look to the target singleton, and avoid being "captured" by the salient abrupt-onset. The unique aspect of this task is there is no physical change in the stimulus at the goal-related location where the target is revealed. In studies that have examined the neural basis of target selection, the entire search array is abruptly presented such that the target or one of the distractors is centered in the RF of a neuron on a given trial (White \& Munoz, 2011a; Shen \& Paré, 2007; McPeek \& Keller, 2002; Schall \& Thompson, 1999). This produces a transient visual response, and target discrimination is typically observed only after this initial nonselective visual burst (Thompson, Hanes, Bichot, \& Schall, 1996). The oculomotor-capture task provides a unique window into the target-directed accumulation process uncontaminated by transient visual activity evoked by abrupt-onset of the array. Using this task, we can better quantify how the goal-related signal at the target location is shaped by competing visual signals elsewhere in the visual field.

We reasoned that the salient abrupt-onset would elicit a high frequency burst of activation on the SCi map that would act to boost the activity of neighboring sites (local excitation; Figure 1E) and suppress activity of distal sites (distal inhibition; Figure 1F). Specifically, we predicted that the visual response evoked by the local abrupt-onset would act to boost activation at the neighboring target location (i.e., boost in baseline; Figure $1 \mathrm{H}$ ) because the excitatory boundaries of their corresponding point images overlap (Figure 1E). As a result, saccades should be initiated earlier (shorter SRT) relative to the control. In contrast, we predicted that the visual response evoked by the remote abrupt-onset would act to suppress activation at the target location (i.e., drop in baseline; Figure 1I) because the boundaries of their corresponding point images do not overlap (Figure $1 \mathrm{~F}$ ). As a result, saccades should be initiated later (longer SRT) relative to the control. In contrast to these predictions, we observed a pattern of presaccadic activation not readily explained by current models of saccade behavior.

\section{METHODS}

Data were collected from two male Rhesus monkeys (Macaca mulatta, monkey $\mathrm{Y}=12 \mathrm{~kg}$, monkey Q $=11 \mathrm{~kg}$ ). The surgical procedures and extracellular recording techniques were detailed previously (Marino et al., 2008) and were approved by the Queen's University Animal Care Committee in accordance with the guidelines of the Canadian Council on Animal Care.

\section{Stimuli and Equipment}

Stimuli were presented on a cathode ray tube monitor at a screen resolution of $1024 \times 768$ pixels $(75 \mathrm{~Hz}$ 


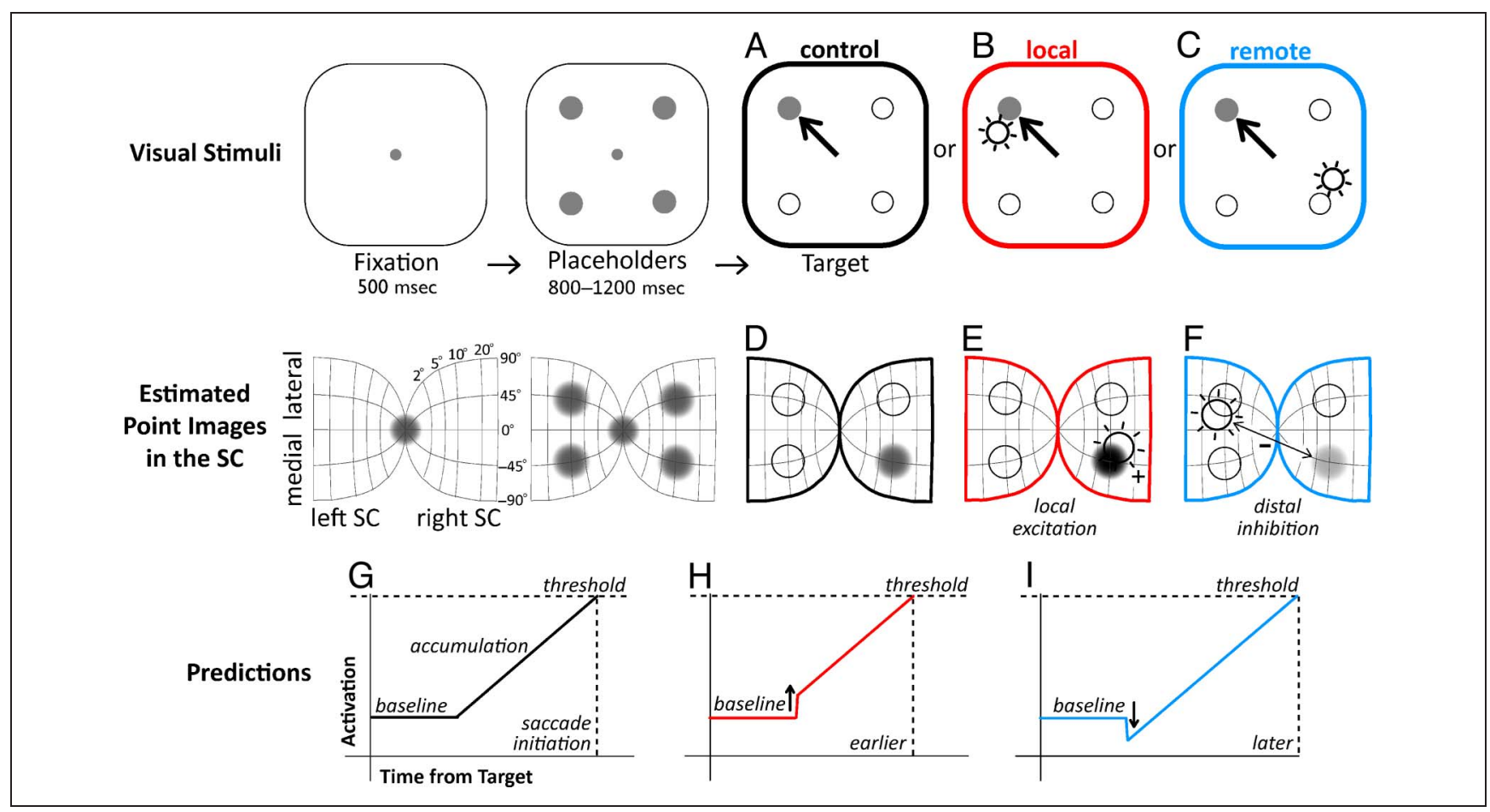

Figure 1. Oculomotor-capture task and estimated point images in the SCi. Monkeys were required to fixate a central fixation stimulus for 500 msec, followed by the onset of a set of homogeneous placeholder stimuli equidistant from fixation (gray $6.5 \mathrm{~cd} / \mathrm{m}^{2}$, against a black background). After an additional delay of 800-1200 msec, the fixation stimulus disappeared, and simultaneously a target singleton was revealed via an isoluminant color change in all but one of the items (three of the items turned red, illustrated here by open circles; A). There was no physical change at the target location. Simultaneous with target appearance on a portion of the trials, an abrupt-onset the same color as the distractors (red) appeared either near (local abrupt-onset; B) or far (remote abrupt-onset; C) from the target goal. The monkeys were required to launch a saccade toward the target singleton for a liquid reward. (D-F) Illustration of the estimated point images (McIlwain, 1986) in the SCi associated with the stimuli in A-C. On the basis of previous estimates (Marino, Trappenberg, et al., 2012; Marino et al., 2008), the point images associated with the local abrupt-onset and the goal overlapped (E), and the point images associated with the remote abrupt-onset and the goal did not overlap (F). (G-I) Illustration of the predictions across the key conditions.

noninterlaced, 8-bit per channel resolution), with a viewing angle of $54^{\circ}$ horizontally and $44^{\circ}$ vertically. The luminance and color properties of the stimuli were measured using the Minolta CS-100 photometer (Minolta, Japan). The behavioral paradigms and visual stimuli were under the control of two Dell 8100 computers running UNIXbased real-time data control and stimulus presentation systems (Rex 6.1; Hays, Richmond, \& Optican, 1982). Eye position was measured using the scleral search coil technique (Robinson, 1963). The data were recorded in a multichannel data acquisition system (Plexon, Inc., Dallas, TX). Eye position and event data were digitized at $1 \mathrm{KHz}$, and action potentials were digitized at $40 \mathrm{kHz}$.

Stimuli were circular disks moderately scaled for eccentricity defined by a given neuron's $\mathrm{RF}$ (from $0.5^{\circ}$ diameter at eccentricity of $3.9^{\circ}$ to $2^{\circ}$ diameter at max eccentricity of $25^{\circ}$ ). Stimulus size never exceeded the RF boundary as confirmed on-line using a RF mapping procedure described previously (White, Boehnke, Marino, Itti, \& Munoz, 2009). All stimuli were presented at $6.5 \mathrm{~cd} / \mathrm{m}^{2}$ against a black background $\left(<0.01 \mathrm{~cd} / \mathrm{m}^{2}\right)$. The fixation point, placeholders, and target were neutral gray (CIE $x=0.29, y=$ 0.28 ), and the distractors including the abrupt-onset item were red (CIE $x=0.64, y=0.33$ ). We used a four-item stimulus array, five items including the abrupt-onset (Figure 1; the distractors and abrupt-onset items are illustrated by open circles). We use the term "distractors" to refer to the three nontarget stimuli that were revealed via an isoluminant color change from the placeholders. We use the term "abrupt-onset" to refer to the additional distractor item, defined by an abrupt luminance increment against the black background. The target and distractors were equally spaced at a $90^{\circ}$ polar angle relative to one another, at equal eccentricity from the fovea, and the entire array was dependent upon the location of the RF for a given neuron. The abrupt-onset appeared at the same eccentricity as the other items, at a polar angle of $\pm 30^{\circ}$ or $\pm 150^{\circ}$ relative to the target, which we refer to as the local versus remote abrupt-onset, respectively. This meant that the abrupt-onset flanked either the target or opposing distractor.

To determine the spatial representation of the stimuli on the map of the $\mathrm{SCi}$, we estimated the corresponding point images (Marino et al., 2008; McIlwain, 1986). A point image refers to the local population of neurons that are activated by a given stimulus. On the basis of estimates of the size of visually evoked point images in the SCi (Marino, Trappenberg, et al., 2012; Marino et al., 2008), two stimuli at equal eccentricity from the fovea that fall within $\sim 60^{\circ}$ polar angle of one 
another will activate overlapping populations of neurons. On the basis of the stimulus configuration in our study, the local abrupt-onset appeared well within this range $\left(30^{\circ}\right.$ polar angle relative to target; Figure $\left.1 \mathrm{E}\right)$, whereas the remote abrupt-onset appeared safely outside this range $\left(150^{\circ}\right.$ polar angle relative to target; Figure $\left.1 \mathrm{~F}\right)$.

\section{Procedure}

During each session, monkeys were seated in a primate chair (Crist Instrument, Hagerstown, MD), in a dark room, head-restrained facing the video monitor. A tungsten microelectrode (0.5-5 M $\Omega$, Frederick Haer, ME) was lowered into the SC while monkeys viewed a dynamic video. The dynamic video served to engage the monkey and generate rich visual stimulation that activated neurons across the SC, making it easier to locate the dorsal surface. Neurons were isolated $1-3 \mathrm{~mm}$ below this point, which represented the approximate locus of the SCi. Once a neuron was isolated, the center of its visual RF was determined using a rapid visual stimulation procedure described previously (White et al., 2009). Monkeys then performed the oculomotor-capture task (Figure 1). Once the monkey fixated the central stimulus for a fixed duration of $500 \mathrm{msec}$, the four placeholder stimuli appeared for 800-1200 msec during which monkeys had to maintain central fixation. The target was then revealed via an isoluminant color change in all but one item (illustrated by the open circles in Figure 1A-F), and the fixation point disappeared at the same time indicating to the monkey to launch a saccade toward the target for a liquid reward. The abrupt-onset, when present, appeared simultaneously with the isoluminant color change that defined the target. A reward was issued only if the saccade endpoint fell within an invisible computer controlled window that was small enough to ensure that the local abrupt-onset did not fall within it. Each trial was followed by a momentary $(800 \mathrm{msec})$ increment in background luminance to prevent dark adaptation. The target appeared with equal probability at any one of the four placeholder locations, such that the target or any of the three distractors had an equal probability of appearing in the RF of the neuron. Also, the local, remote, and no abrupt-onset conditions occurred with equal probability. This resulted in 12 primary conditions (3 Abruptonset conditions $\times 4$ Target locations), all of which were randomly interleaved.

\section{Analyses}

A saccade was defined as an eye movement that exceeded a velocity criterion $\left(35^{\circ} / \mathrm{sec}\right)$. Only the first saccade after target appearance was analyzed. SRT was the time from target appearance to saccade onset. Saccades with SRT of $<60$ msec or $>500$ msec were excluded, which resulted in removal of less than $0.1 \%$ of trials across 46 sessions. A saccade was considered correct if its endpoint fell within the computer controlled window surrounding the target (described earlier) and was closer to the target in Euclidean distance than any of the other items. Otherwise, the saccade was considered a direction error. Because there were so few direction errors ( $<4 \%$ on average), analysis was focused on correct trials only. We obtained at least 10 correct trials per condition for each target location in a given session.

Single units were isolated on-line using a window discriminator and confirmed off-line using spike sorting software (Plexon, Inc.). Spike density functions were created by convolving individual spikes with a Gaussian kernel $(\sigma=$ $5 \mathrm{msec}$ ). For population analyses, firing rates for each neuron were normalized to the maximum response of that neuron. Averages and statistics were computed from these normalized values. For the purpose of this study, neurons were isolated approximately $1-3 \mathrm{~mm}$ below the first sign of entering the SC, which represented the approximate locus of the SCi. We recorded extracellular activity of $46 \mathrm{SCi}$ neurons (20 from monkey Y, 26 from monkey Q; one neuron per session). All the neurons were classified as visuomotor. Neurons had a visual component if the average activity immediately following the onset of the placeholder stimuli (40-120 msec) was significantly greater than the average activation over a prestimulus reference period $(-80 \mathrm{msec}$ to array onset; $p<.05$ across all neurons, $t$ test). Visual response latency was defined as the point where this activation first exceeded three standard deviations above the prestimulus reference period and remained so for at least 10 msec. Neurons had a motor component if the average firing rate around the time of the saccade $( \pm 25$ msec relative to saccade onset) was significantly greater for saccades directed into versus opposite its $\mathrm{RF}(p<.00001$ across all neurons, $t$ test).

We refer to the goal-related response as the activation profile associated with correctly directed saccades toward the target starting from target appearance to the time of saccade initiation. We estimated differences between conditions in terms of, (i) baseline activation, (ii) accumulation rate (i.e., rate of rise) of the goal-related response in both the target-aligned and saccade-aligned case, and (iii) saccade threshold activation level.

Generally speaking, baseline refers to some level of activation before target-directed accumulation begins (Carpenter, 1988). We defined the transient suppression as a change (i.e., drop) in baseline because the activation momentarily fell below what naturally occurred in the absence of a salient abrupt-onset (i.e., the control condition), immediately before the target-directed activation began to accumulate toward threshold. Thus, for each neuron, we determined an epoch (Epoch baseline $_{\text {) }}$ that best captured the period of transient suppression evoked by the local abrupt-onset (see gray bar in Figure 5A). We did so by first computing the time of the trough of the suppression (suppression latency). Because target-directed activation naturally began rising shortly after target appearance, we defined the trough of the suppression as the point of lowest discharge starting from the time of target appearance plus the neuron's visual onset latency (defined 
earlier), to the mean SRT for the session. Epoch baseline was $_{\text {a }}$ then defined as a $\pm 10 \mathrm{msec}$ window centered on this point. There was no clear suppression associated with the remote abrupt-onset condition, so Epoch baseline was used to also make comparisons with this condition. Suppression magnitude was defined as the percentage of decrease of the activation level during Epoch $_{\text {baseline }}$ for the local abrupt-onset condition relative to the control.

Second, for each neuron we estimated the accumulation rate (rate of rise) of the goal-related response (see Figure $5 \mathrm{~A}, \mathrm{H})$. This was achieved by fitting a least-squares regression line to the goal-related activation profile over a $75-\mathrm{msec}$ epoch and calculating the slope (spks/sec/msec). This was computed for data aligned on target and saccade onset. For the target-aligned case, this epoch started at the trough of the transient suppression for a given neuron (defined earlier), which represented the point where activation reversed and first began to increase toward threshold. The 75-msec epoch was partly imposed by the data because we wanted to ensure that it was less than the interval between the suppression trough and saccade onset, and the trial vectors had to be the same length because they were averaged within a given condition of a neuron. For the saccade-aligned case, this epoch started at -100 msec relative to saccade onset and then continued for the same duration as the target-aligned case (75 msec), ending just before the interval defining threshold. To perform within-neuron correlations between accumulation rate and SRT, we had to obtain an estimate of accumulation rate for each trial. We expected this estimate to be less reliable, so for each trial we chose an interval starting from the trough of the suppression estimated earlier to $-25 \mathrm{msec}$ relative to saccade onset. Thus, this size of this interval was often greater than $75 \mathrm{msec}$ and varied depending on SRT for that trial. Although somewhat different from the method used earlier, it yielded reliable estimates of the trial-by-trial accumulation rate necessary for within-neuron correlations.

Finally, we estimated saccade threshold activation by computing the average discharge rate over a 10-msec epoch from 18 to $8 \mathrm{msec}$ before saccade onset $\left(\mathrm{Epoch}_{\text {threshold; }}\right.$ see gray bar in Figure $5 \mathrm{H}$ ). The rationale for this epoch was based on physiological reports that the shortest time a saccade can be influenced by a neuronal signal from the SCi falls within this range (Miyashita \& Hikosaka, 1996; Munoz, Waitzman, \& Wurtz, 1996). This estimate was also chosen in light of a similar but slightly earlier estimate for FEF of 20-10 msec before saccade onset (Purcell et al., 2012; Hanes \& Schall, 1996).

\section{RESULTS}

\section{Behavior}

\section{Saccade Direction Errors}

For untrained human participants, the oculomotor-capture task can elicit many saccade direction errors-roughly
30-40\% (Theeuwes et al., 1999). Here, monkeys performed comparatively better, most likely because of the high degree of training required for monkeys to learn the rules of the task. Figure 2A shows the cumulative distribution of errors across the 46 sessions for the key conditions. Overall, there were less than $12 \%$ errors across all sessions. Figure 2B shows the mean percentage of errors (triangles and squares represent the data from monkey $\mathrm{Y}$ and Q, respectively). Although monkey Q made visibly fewer errors than monkey $\mathrm{Y}$, the pattern in the abruptonset conditions was the same, so we collapsed the data and performed a repeated-measures ANOVA across the conditions. The ANOVA revealed a significant difference in Error Rates, $F(4,180)=4.96, p<.001$. Bonferronicorrected post hoc tests revealed a significantly greater percentage of errors directed toward the local (red bars) versus remote (blue bars) abrupt-onset, $t(45)=3.12$, $p=.003$, indicating greater competition from abruptonsets that appeared closest to the goal. Also, there were significantly more errors directed toward the opposite distractor relative to the ipsilateral, $t(45)=2.8, p=.007$, and contralateral, $t(45)=2.3, p=.02$, distractors, but this was primarily driven by the data obtained from one monkey (Y).

\section{$S R T$}

The hypotheses outlined in this study emphasize SRT as a key behavioral index of the spatial competition between visual and goal-related signals. We predicted that the local abrupt-onset would elicit shorter SRTs, whereas the remote abrupt-onset would elicit prolonged SRTs. The behavioral results were in line with these predictions. Figure 2C shows the cumulative distribution of mean SRTs across the 46 sessions for the key conditions. Figure 2D shows the mean SRTs between these conditions (triangles and squares represent the data from monkey $\mathrm{Y}$ and $\mathrm{Q}$, respectively). For both monkeys, the trend was similar, so we collapsed the data and ran a repeated-measured ANOVA across the conditions. The ANOVA revealed a significant difference in SRT across the conditions, $F(2,90)=42.3, p<.001$. Bonferroni corrected post hoc tests revealed that SRTs were shorter in the local abrupt-onset condition relative to the no abrupt-onset control condition, $t(45)=6.7, p<.001$. This is in line with the hypothesis that the overlapping visual and goal-related signals associated with this condition were mutually excitatory (Figure 1B), thereby elevating baseline activation closer to saccade threshold. In contrast, SRTs were prolonged in the remote abruptonset condition relative to the no abrupt-onset control condition, $t(45)=3.2, p=.002$. This is in line with the hypothesis that the nonoverlapping visual and goalrelated signals associated with this condition were mutually inhibitory (Figure 1C), thereby suppressing baseline activation away from saccade threshold. This latter result is also consistent with human studies on the effect of remote 
Figure 2. Summary of behavior on the oculomotorcapture task. (A and B) Percentage of saccade direction errors across all sessions. (C and D) SRT for correctly directed saccades. Squares and triangles represent the results of monkey Q and Y, respectively. Ipsi, contra, and opp D refer to the distractor items that were ipsilateral, contralateral, or opposite the target location, respectively.

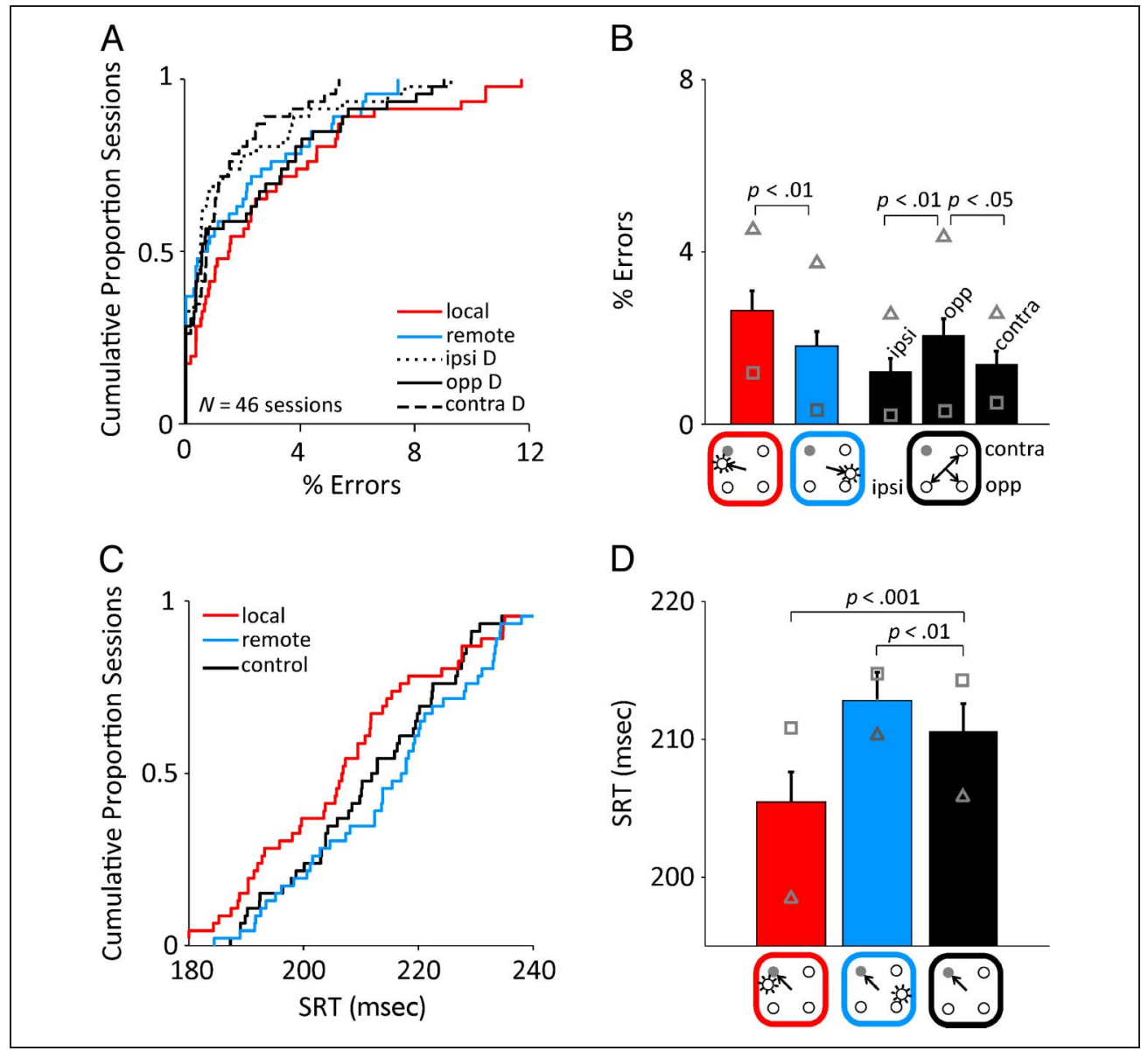

distractors (McSorley, McCloy, \& Lyne, 2012; Bompas \& Sumner, 2011; Born \& Kerzel, 2011; White, Gegenfurtner, \& Kerzel, 2005; Walker, Deubel, Schneider, \& Findlay, 1997).

\section{Neuronal Activation}

Characteristics of the Neuronal Response during the Oculomotor-capture Task

We recorded extracellular single-unit activity from 46 visuomotor neurons in the SCi of two monkeys (20 from monkey Y, 26 from monkey Q) that performed the oculomotor-capture task. Figure 3 shows a single unit example across the key conditions. First, the onset of the array of placeholders produced a characteristic transient visual response because one of the items appeared in the neuron's RF (Figure 3A). This was followed by a sustained response that gradually ramped up as target appearance approached (Figure 3B). Most of the neurons (42/46, 91\%) showed a significant increase in the mean discharge rate leading up to target appearance $(p<$ .05 , repeated-measures ANOVA across 100-msec intervals from $-500 \mathrm{msec}$ to target appearance). This suggests that most of the neurons were like the "prelude" or "buildup" type described previously (Munoz \& Wurtz, 1995; Glimcher \& Sparks, 1992), which are commonly associated with target selection (Keller \& McPeek, 2002). Once the target was revealed, activation quickly increased when the target was in the RF of the neuron (Figure 3B) and quickly decreased when a distractor was in the RF of the neuron (Figure 3C). Finally, there was a high-frequency burst of activation around the time saccades were elicited in the direction of the RF (Figure 3D).

Because the target was revealed via an isoluminant color change at the nontarget locations, there was no physical change associated with the target stimulus. This allowed us to compare pure modulation of the goalrelated response across stimulus conditions as the target selection process developed. When the target was in the $\mathrm{RF}$ and the local abrupt-onset was present (Figure 3B, red line), we observed a transient decrease in discharge rate (essentially a drop in baseline activation highlighted by the downward arrow) relative to the control condition (black line). This transient decrease was time-locked to the appearance of the local abrupt-onset because it was absent when the data were aligned on saccade onset (Figure $3 \mathrm{D}$, red line). In contrast to our prediction, this is indicative of spatial competition, which suggests that the point images associated with the local abrupt-onset and the goal were nonoverlapping. However, rather than delay the saccadic response, this momentary suppression was followed by a rapid rebound in goal-related activation 
(essentially greater accumulation rate highlighted by the upward arrow), which quickly exceeded the other conditions, and was associated with shorter target-directed SRTs.

A similar transient suppression was observed at the distractor locations (Figure 3C, highlighted by the downward arrow). Although the decrease in activation at the distractor-related sites is characteristic of the selection process, it is notable how sharply the activation dropped and how closely it resembled the suppression at the target-related site in the local abrupt-onset condition. Following this transient decrease in the distractor case, we observed a pronounced rebound (highlighted by the upward arrow) for distractors that were flanked by the remote abrupt-onset (Figure 3C, blue line). This is indicative of competition from the remote abrupt-onset and is consistent with the slower SRTs associated with remote distractors (Figure 2C-D; McSorley et al., 2012; Bompas \& Sumner, 2011; Born \& Kerzel, 2011; White et al., 2005; Walker et al., 1997).
Figure 4 summarizes the latency and magnitude of the transient suppression. Suppression latency was defined as the time of the trough of the suppression, and suppression magnitude was defined as the percentage of decrease relative to the control condition (see Methods). Across the 46 neurons, the suppression latency (median $86 \mathrm{msec}$ ) occurred reliably after visual response latencies (median 49 msec; Figure 4A). The box and whiskers represent $75 \%$ and 99\% confidence intervals, respectively. In the following sections, we quantify these observations statistically.

\section{Between-condition Comparison of Baseline, Threshold, and Accumulation Rate of the Goal-directed Signal}

The primary aim of this study was to determine how the goal-related signal is shaped by its spatial proximity to a salient abrupt-onset. To do so, we obtained estimates of three important parameters of the goal-related signal

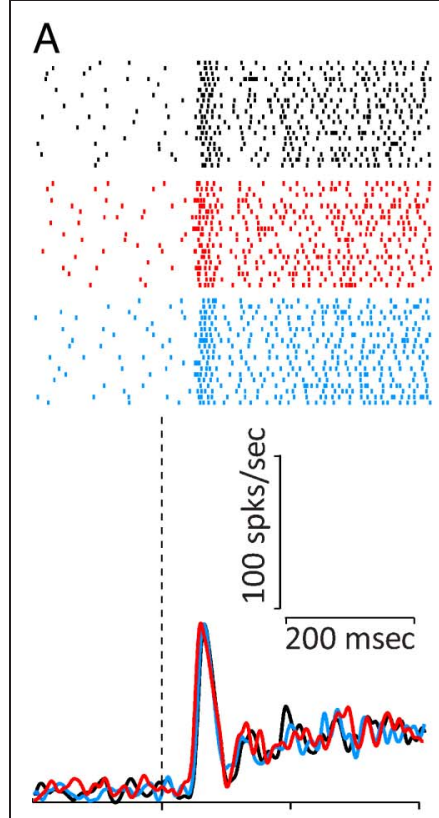

Time From Array (msec)

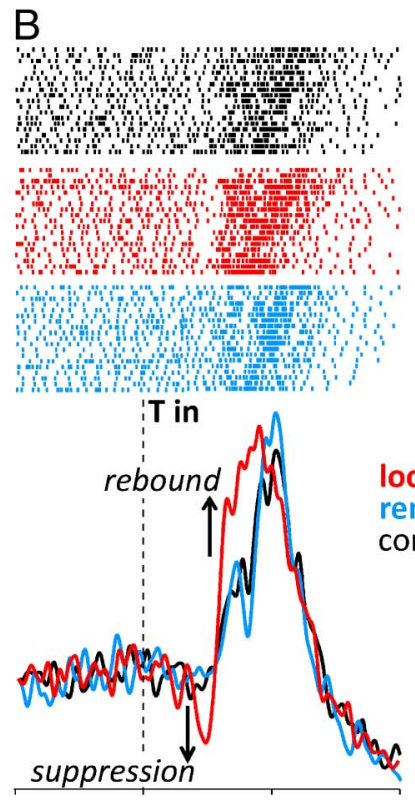

Time From Target (msec)

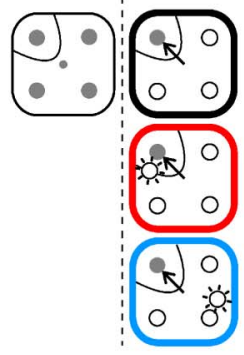

C

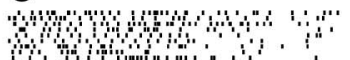

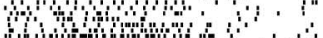
and ? (n)

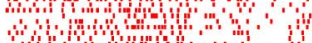
nhand .4. and mond $D$ in

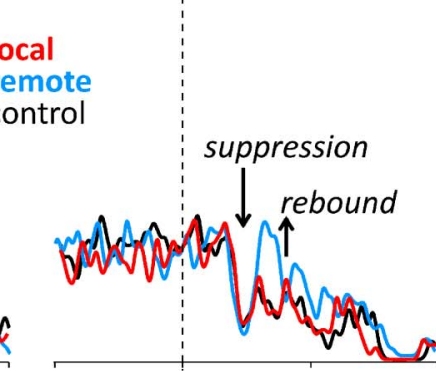

Time From Target (msec)

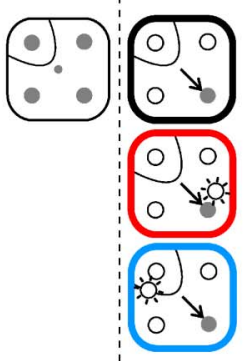

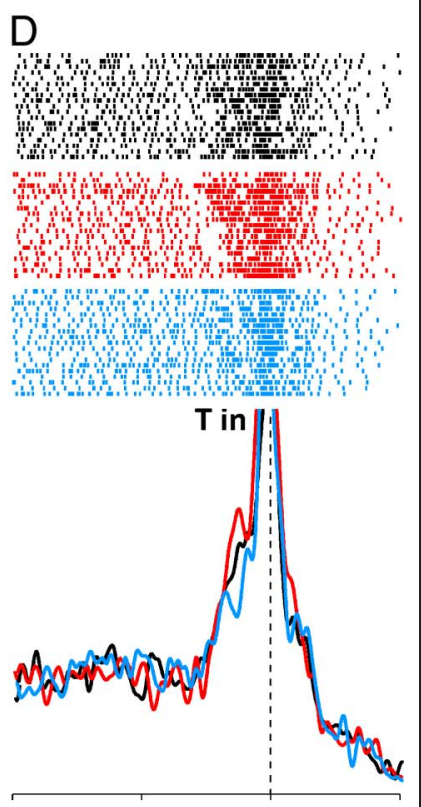

Time From Saccade (msec)

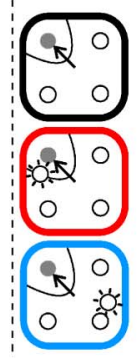

Figure 3. Characteristics of a single-unit response across stimulus conditions. (A) The visual response evoked from the onset of the array of homogenous placeholders. (B) Activation leading up to and following the appearance of the target in the RF of the neuron for each condition ( $\mathrm{T}$ in = target in the RF). (C) Activation leading up to and following the appearance of the distractor in the RF of the neuron for each condition (D in = distractor in the RF). Only activation associated with the opposite distractor is shown for brevity. (D) Activation leading up to and following the saccade into the RF of the neuron for each condition (spike density function in saccade-aligned case truncated to fit within the range of the ordinate). The illustrations depict the visual display across conditions, with the upper left item centered in the RF of the neuron. 
Figure 4. Characteristics of the transient suppression. (A and B) Cumulative distributions of the latency and magnitude of the transient suppression across the 46 SCi neurons. Visual response latency (dotted line) is plotted for comparison and was derived from the visual responses evoked by the onset of the array of placeholders at the beginning of the trial (Figure $3 \mathrm{~A}$ ). The box and whiskers of the boxplots represent the $75 \%$ and $99 \%$ confidence intervals, respectively. The thick line at the center of the boxplots represents the median.

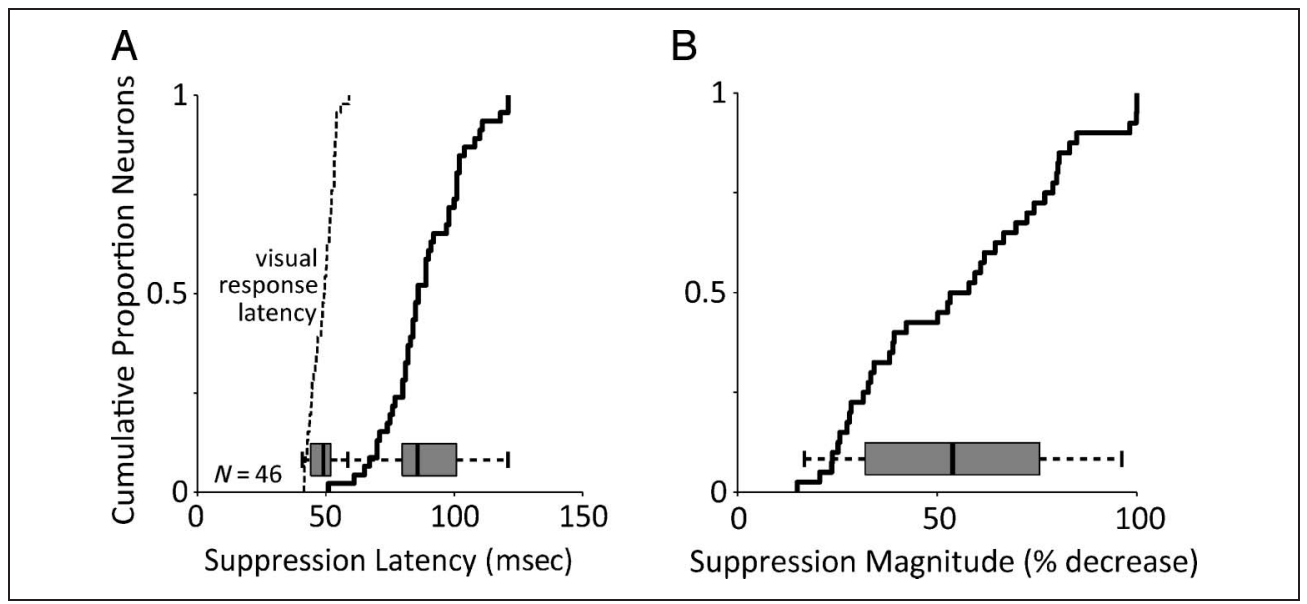

specified by accumulator models (see Methods): We estimated differences between conditions in terms of (i) the baseline activation following target appearance

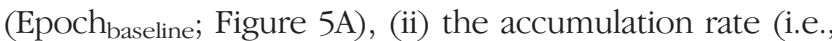
rate of rise) of the goal-related signal in both the targetaligned (Figure 5A) and saccade-aligned (Figure 5H) case, and (iii) the saccade threshold activation level $\left(\right.$ Epoch $_{\text {threshold; }}$ Figure $5 \mathrm{H}$ ).

The differences described earlier in the single unit were evident in the population averages (Figure $5 \mathrm{~A}, \mathrm{H}$ ). Figure 5B, D, and F compared the estimate of the change

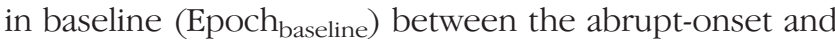
control conditions. In Figure 5B, most of the neurons (87\%) fell below the line of unity, indicating lower activation for the local abrupt-onset condition relative to the

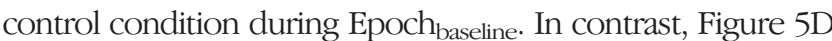
showed little difference in activation between the remote abrupt-onset condition and the control condition during

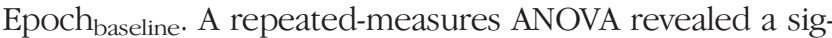
nificant difference in activation across conditions during Epoch $_{\text {baseline, }} F(2,90)=31.2, p<.001$. Bonferroni corrected post hoc tests confirmed that there was significantly lower activation in the local abrupt-onset condition relative to the control condition, $t(45)=6.3, p<.001$, but no difference between the remote abrupt-onset condition relative to the control condition, $t(45)=1.4, p=.16$. These results indicate that only the local abrupt-onset condition induced a reliable suppression in baseline activation (Figure $5 \mathrm{~F}$ ).

The pattern in terms of accumulation rate was opposite to the pattern in terms of baseline. Figure $5 \mathrm{C}, \mathrm{E}$, and $\mathrm{G}$ compared the estimate of accumulation rate between the abrupt-onset and control conditions. In Figure 5C, most of the neurons (83\%) fell above the line of unity, indicating greater accumulation rate (i.e., faster rate of rise) of the goal-related signal for the local abrupt-onset condition relative to the control condition. In contrast, Figure 5E showed little difference in accumulation rate between the remote abrupt-onset condition and the control condition. A repeated-measures ANOVA revealed a significant difference in Accumulation Rate across the conditions, $F(2,90)=34.0, p<.001$. Bonferroni corrected post hoc tests confirmed that Accumulation Rate was significantly greater in the local abrupt-onset condition relative to the control condition, $t(45)=6.2, p<$ .001 , but was not different between the remote abruptonset condition and the control condition, $t(45)=0.1$, $p=.91$ (Figure 5G). Taken together, these results indicate that although the local abrupt-onset induced a robust transient decrease in goal-related activation (essentially a drop in the baseline), it was also associated with a rebound that resulted in a greater accumulation rate of goal-related activation, which induced the shorter SRTs associated with this condition.

The same analysis was performed on the data aligned on saccade onset ${ }^{1}$ (Figure $5 \mathrm{H}-\mathrm{N}$ ). There was a significant difference in Accumulation Rate across the conditions, $F(2$, $90)=18.16, p<.001$. As in the target-aligned case, Bonferroni corrected post hoc tests confirmed that accumulation rate was significantly greater in the local abrupt-onset condition relative to the control condition, $t(45)=4.2, p<.001$, but was not different between the remote abrupt-onset condition and the control condition, $t(45)=0.4, p=.66$ (Figure 5I, K, M).

Finally, Figure 5J, L, and N compared the estimate of saccade threshold activation (Epoch threshold $_{\text {) between }}$ the abrupt-onset and control conditions. Figure 5J and L showed approximately the same percentage of neurons above or below the line of unity, suggesting little differences in threshold activation between conditions. A repeated-measures ANOVA revealed no significant differ-

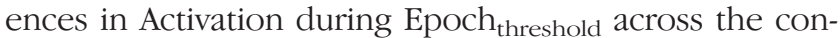
ditions, $F(2,90)=2.6, p>.05$. Thus, differences in saccade threshold cannot adequately account for the difference in SRT between conditions. Rather, it is the difference in accumulation rate that best accounts for the differences in SRT associated with the stimulus conditions. This is consistent with results obtained previously in the FEF (Hanes \& Schall, 1996) and the SC (Paré \& Hanes, 2003). 


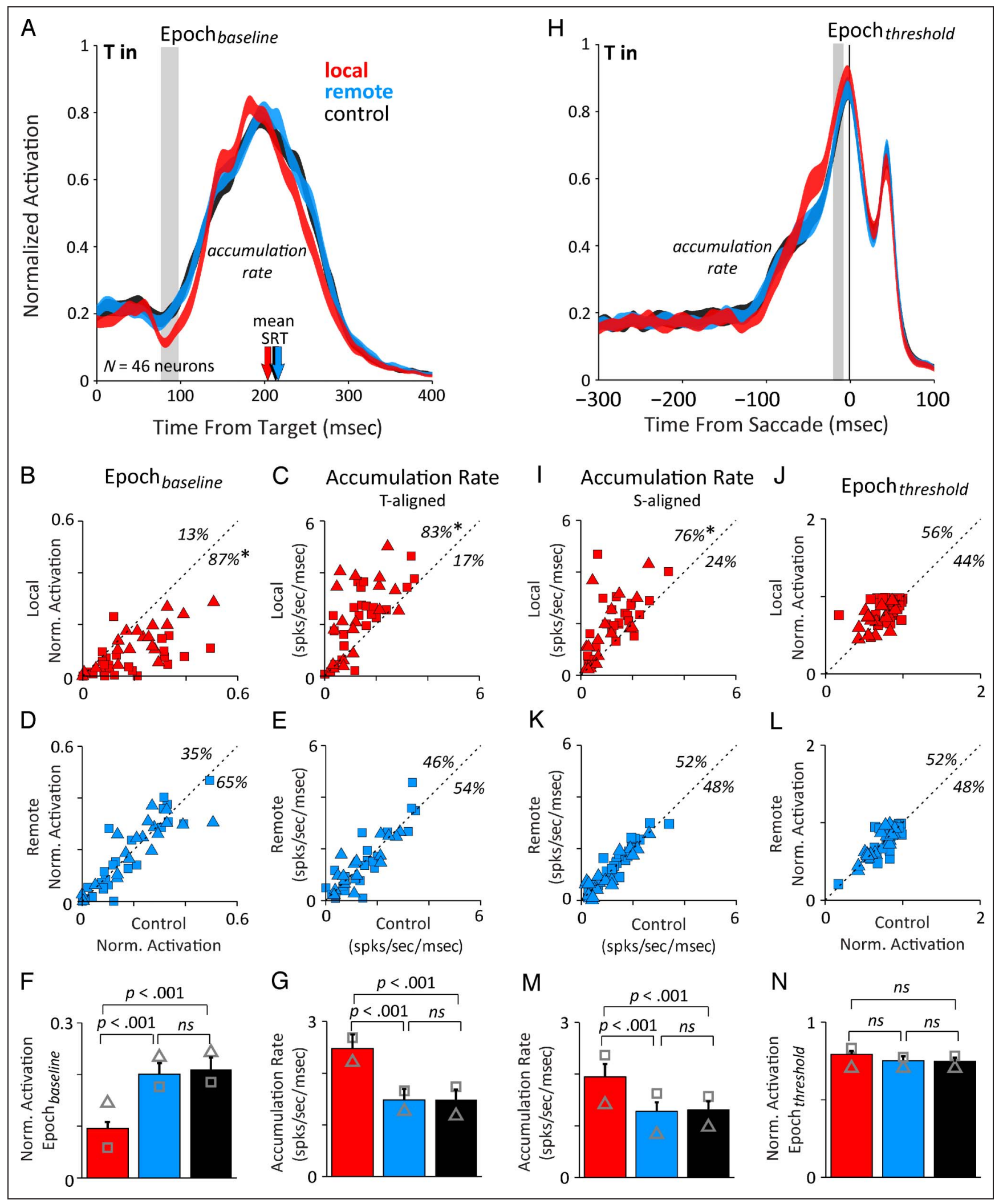

Figure 5. Comparison of baseline, threshold, and accumulation rate of the goal-related signal. (A) Normalized population average responses $( \pm S E M)$ aligned on target appearance (target in the RF). (B and D) Comparison of activation level during Epoch baseline $(\mathrm{estimate}$ of baseline, see Methods). (C and E) Comparison of accumulation rate of the goal-related signal (target aligned). Squares and triangles represent the data from monkey Q and Y, respectively. (F and G) Summary of the differences. (H) Normalized population average responses aligned on saccade onset. (I and K) Comparison of the accumulation rate of the goal-related signal (saccade aligned). ( $\mathrm{J}$ and $\mathrm{L}$ ) Comparison of activation level during Epoch $_{\text {threshold }}$ (estimate of threshold). (M and N) Summary of the differences. 
Between-condition Comparison of

Distractor-evoked Activation

Recall in the single unit example (Figure 3C) there was evidence of an early transient suppression at the opposite distractor location, followed by a pronounced rebound when flanked by a remote abrupt-onset. Here we quantify these observations across the population. Figure 6A plots normalized distractor-evoked population average responses across the key conditions (opposite distractor in the RF; target aligned). We observed a small visual response (indicated by the arrow in Figure 6A), which was associated with the isoluminant color change that defined the distractors. This is consistent with isoluminant color responses in the SC (White et al., 2009). This initial visual response was followed by a fairly distinct dip in activation that coincided closely with the interval associated with the transient suppression described earlier (note the shaded region representing Epoch baseline in Figure 6A). Because distractor-evoked activation should naturally decrease as a result of the target selection process, we reasoned that an increase in distractor-evoked

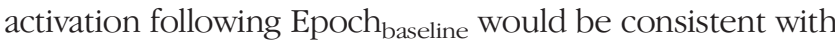
the idea that the dip represents suppression similar to that observed in the target-related case (Figure 5A). We com-

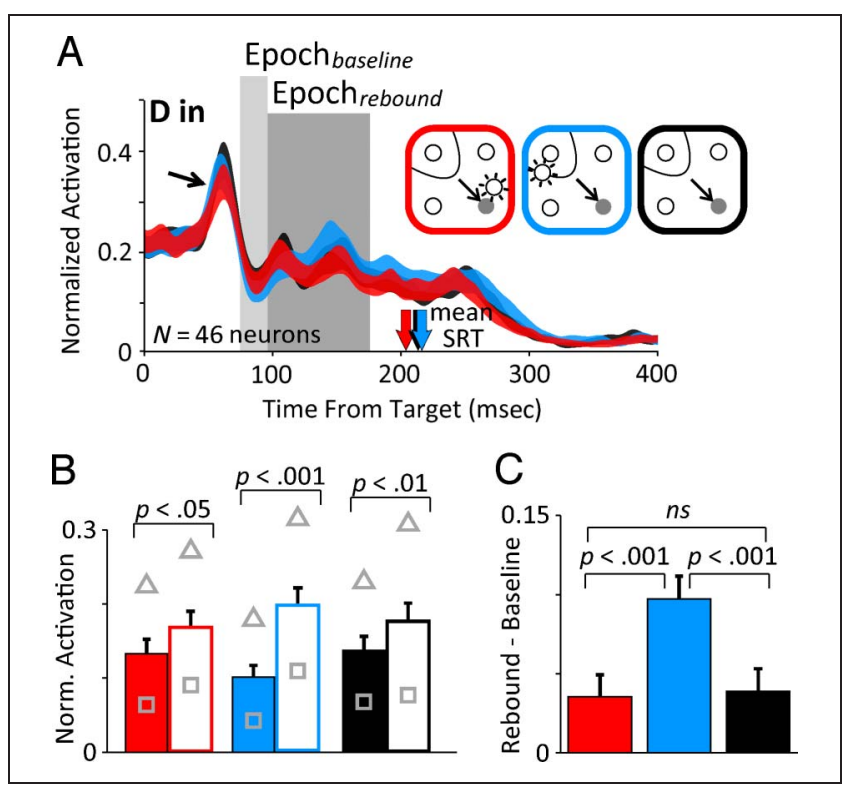

Figure 6. Distractor-related activation. (A) Normalized population average responses $( \pm S E M)$ aligned on target appearance (opposite distractor in the RF; note the illustrations in the inset). The light shaded region $\left(\right.$ Epoch $_{\text {baseline }}$ ) represents the epoch associated with the transient suppression in Figure 5. The darker shaded region (Epoch rebound) represents the interval associated with the target-related rebound described earlier (i.e., the epoch over which accumulation rate was estimated in Figure 5A). (B) Compares the average distractor-evoked

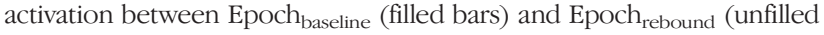
bars) across the key conditions. (C) Plots the differences between $\mathrm{Epoch}_{\text {baseline }}$ and $\mathrm{Epoch}_{\text {rebound, with positive values indicating greater }}$ activation for the latter. pared distractor-evoked activation between Epoch $_{\text {baseline }}$ and subsequent Epoch $_{\text {rebound }}$, defined as the same interval associated with the target-related rebound described earlier (i.e., the 75-msec epoch over which accumulation rate was estimated in Figure 5A). Figure 6B shows mean distractor-evoked activation during Epoch baseline $_{\text {(filled }}$ bars) and Epoch ${ }_{\text {rebound }}$ (unfilled bars) across the key conditions. We performed a $2 \times 3$ (Epoch $\times$ Abrupt-onset condition) repeated-measures ANOVA on the distractorevoked activation. The ANOVA revealed a main effect of Epoch, $F(1,45)=22.08, p<.001$, and a significant interaction, $F(2,90)=15.63, p<.001$. Bonferroni corrected post hoc comparisons revealed that distractor-evoked activation was lower during Epoch baseline $_{\text {than }}$ the subsequent Epoch ${ }_{\text {rebound }}, t(45)=4.69, p<.001$. In addition, the difference was greater in the remote abrupt-onset condition (Figure 6C) than the local abrupt-onset condition, $t(45)=4.42, p<.001$, or the control condition, $t(45)=$ $4.57, p<.001$. This result is in alignment with the longer SRTs associated with the remote abrupt-onset condition (Figure 2C, D). The suppression was also observed at ipsilateral, but not contralateral, distractor locations (not shown), and when the abrupt-onset was absent. Taken together, this implies that the suppression occurred at most locations associated with the common feature defining the distractors and abrupt-onsets (i.e., the color red). This is reminiscent of the type of experience-induced feature suppression built up through extensive training (Bichot, Schall, \& Thompson, 1996).

\section{Within-neuron Correlation between Goal-directed Accumulation Rate and SRT}

Our results showed that the shorter average SRT in the local abrupt-onset condition was associated with a greater average accumulation rate of the goal-related signal (Figure 5C, I). To establish that SRT was directly related to the trial-bytrial variation in accumulation rate in our study, we performed within-neuron correlations between these two factors. To do so, we estimated the trial-by-trial accumulation rate (see Methods). Figure 7A, C, and E show a single unit example for the local, remote, and no abrupt-onset conditions, respectively. Each point represents a single trial. Across the three conditions, one can see a negative relationship between accumulation rate and SRT (i.e., shorter SRT is associated with greater accumulation rate). Figure $7 \mathrm{~B}, \mathrm{D}$, and $\mathrm{F}$ show the distribution of correlation coefficients (Spearman's $R$ ) across the population of 46 SCi neurons (gray bars represent the neurons showing a significant negative correlation, $p<.05$ ). Although only $30 \%$ (14/46, control condition) to 36\% (17/46, abrupt-onset conditions) of neurons showed a statistically significant negative correlation between accumulation rate and SRT (gray bars), the distributions of correlation coefficients were reliably shifted leftward across all conditions indicating a negative relationship, and this shift was statistically significant $(p<.001$ across all conditions, Wilcoxon 
signed-rank test for zero median). It should be noted that the $R$ values are not an indicator of the magnitude of the slope of the relationship. These results simply confirm that SRT was directly related to the trial-by-trial accumulation rate. The slope of this relationship was still greatest in the local abrupt-onset condition, as seen in the single unit example (Figure 7A, C, and E) and as shown previously in Figure 5.

\section{Relationship between the Transient Suppression and Goal-directed Accumulation Rate}

Because the transient suppression was immediately followed by a rebound that facilitated saccade initiation, it raised the question whether the two processes are associated with a common mechanism. Therefore, we examined the relationship between the trial-by-trial estimate of accumulation rate (derived earlier) and a trial-by-trial estimate of suppression magnitude. We estimated the trial-by-trial suppression magnitude by computing the percentage decrease of the activation

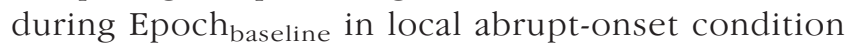
for each trial from the mean activation level during Epoch $_{\text {baseline in the control condition. Figure 8A shows }}$ the correlation between the trial-by-trial suppression magnitude and accumulation rate for an example neuron. Figure 8B shows the distribution of correlation coefficients (Spearman's R) across the 46 neurons. Although
Figure 7. Within-neuron correlation between goalrelated accumulation rate and SRT. (A, C, and E) Spearman's correlations between the trial-by-trial accumulation rate and SRT for the local, remote, and no abrupt-onset conditions $(R=$ correlation coefficient, $m=$ slope of the relationship). (B, D, and $\mathrm{F}$ ) The distributions of the correlation coefficients for the $46 \mathrm{SCi}$ neurons. Gray bars represent individual neurons that showed a significant correlation between SRT and accumulation rate. The $z$ and $p$ values in $\mathrm{B}, \mathrm{D}$, and $\mathrm{F}$ refer to the results of a Wilcoxon signed-rank test for zero median for the distributions of correlation coefficients.

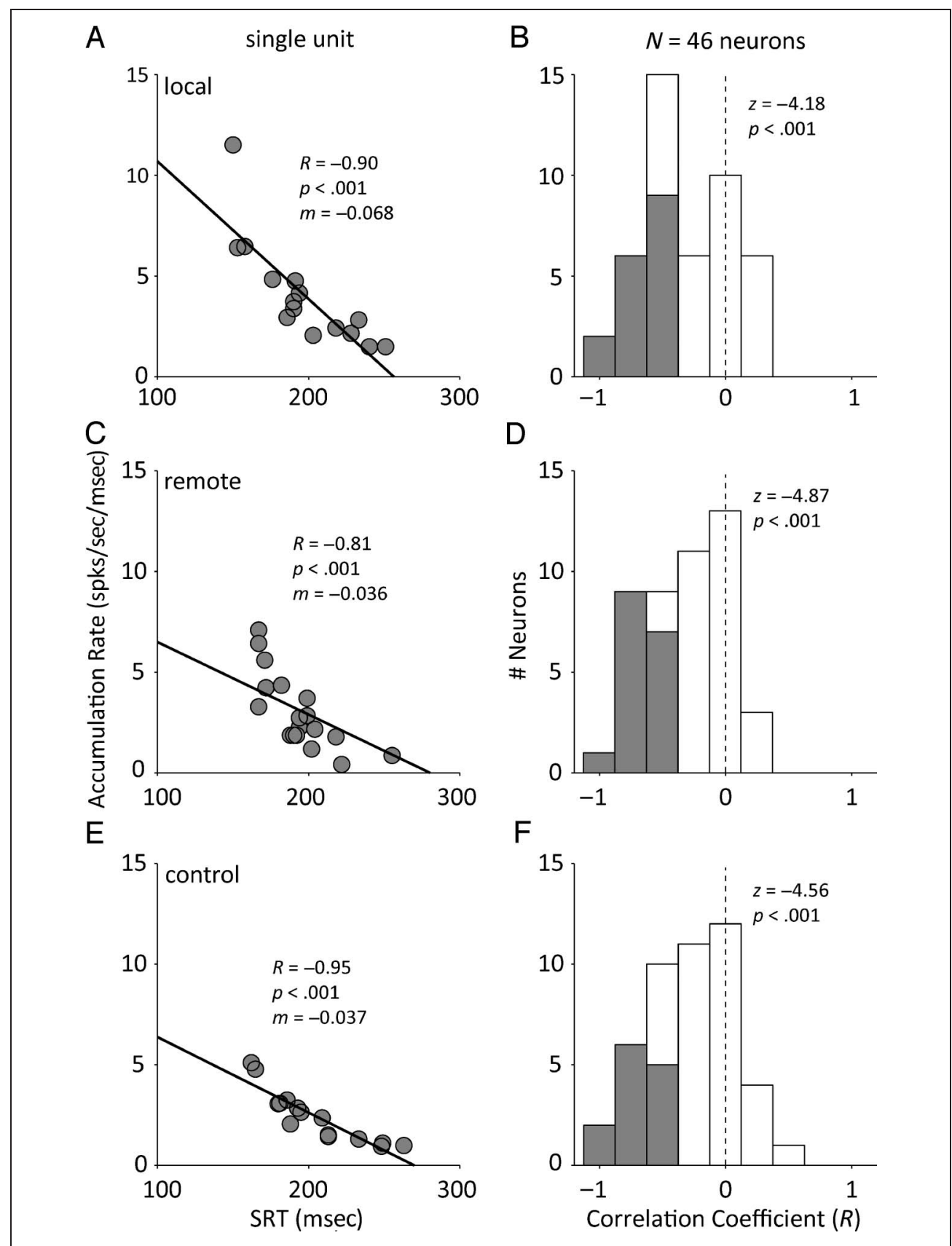


Figure 8. Within-neuron correlation between suppression magnitude and accumulation rate. (A) The Spearman's correlation between the trial-by-trial suppression magnitude and accumulation rate for a single neuron. (B) The distribution of correlation coefficients for the 46 SCi neurons. Gray bars represent individual neurons that showed a significant correlation between suppression magnitude and accumulation rate. The $z$ and $p$ values in $\mathrm{B}$ refer to a Wilcoxon signed-rank test for zero median for the distribution. (C) Normalized population average responses $( \pm S E M)$ in which the trials within each neuron were separated by the median split in accumulation rate ( $N=46$ neurons, local abrupt-onset condition). Black is associated with the faster accumulation rate, and gray is associated with the slower accumulation rate. (D) The suppression magnitude associated with the slower versus faster accumulation rate. The $z$ and $p$ values in $\mathrm{D}$ refer to a Wilcoxon paired-samples test.
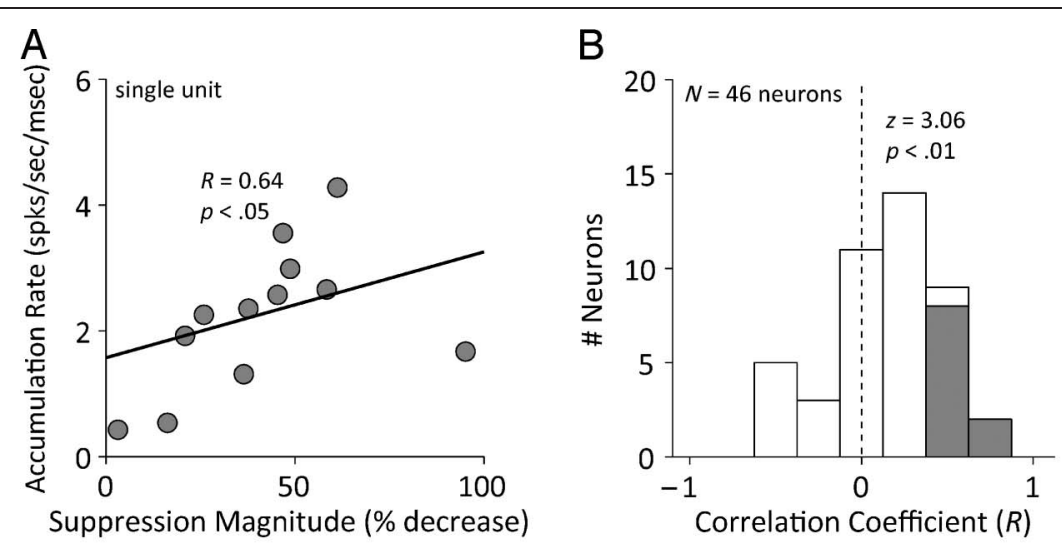

C

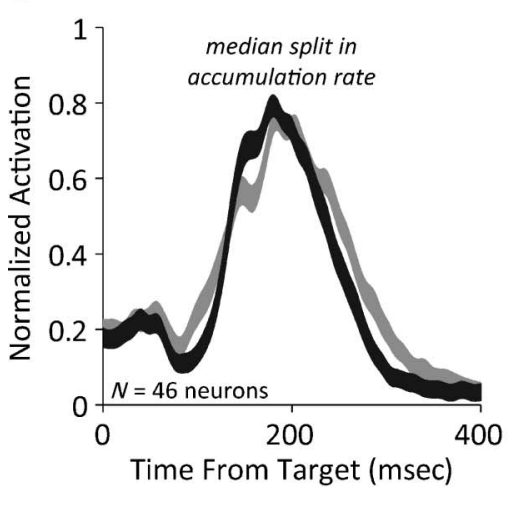

D

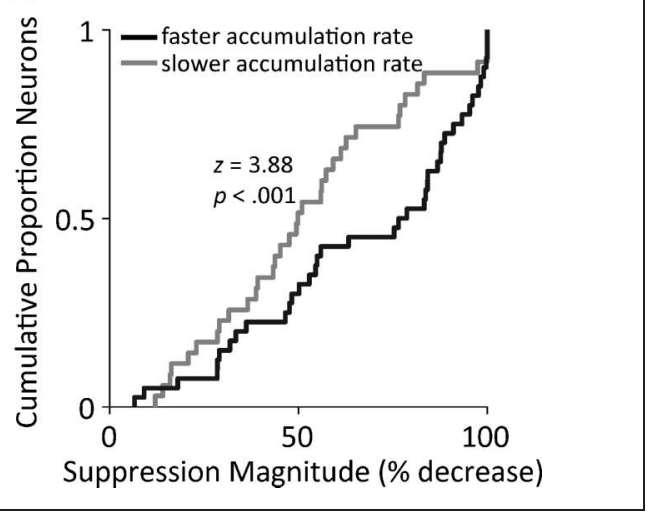

the relationship was modest, there was a clear trend in the positive direction with 10/46 (22\%) neurons showing a significant positive correlation between suppression magnitude and accumulation rate $(p<.05)$. Also, the distribution of correlation coefficients was shifted to the right of zero indicating a positive relationship, which was statistically significant $(z=3.06, p<$ .01, Wilcoxon signed-rank test for zero median). Taking a reverse approach, we divided the trials within each neuron according to the median split in accumulation rate and then measured the magnitude of the suppression in the slower versus faster accumulation rate bins. Figure 8C shows population-averaged spike density functions associated with slower versus faster accumulation rate (target in $\mathrm{RF}$, local abrupt-onset condition). Again, faster accumulation rate was associated with greater suppression magnitude (Figure 8D, $z=3.88$, $p<.001$, Wilcoxon paired-samples test). This confirms that at least some of the variation in accumulation rate is related to the earlier suppression. Taken together, these results establish a link between the magnitude of the transient suppression, the subsequent rebound in accumulation of neuronal activity, and subsequent saccadic behavior.

\section{DISCUSSION}

This study utilized the oculomotor-capture task (Theeuwes et al., 1999) to explore how the spatial proximity between competing visual and goal-related signals shapes the accumulation of goal-directed activity. The unique aspect of this task was that there was no physical change in the stimulus defining the target, allowing us to precisely quantify modulations of the goal-directed response as a result of competing visual signals. We quantified differences in the goal-directed discharge of SCi neurons in terms of three basic parameters of accumulator models: baseline, accumulation rate, and threshold. We predicted that an abrupt-onset near the target would act to boost goal-directed activation (i.e., boost in baseline; Figure $1 \mathrm{H}$ ) because the excitatory boundaries of their corresponding point images overlap (Figure 1E). In contrast, we predicted that an abrupt-onset far from the target would act to suppress goal-directed activation (i.e., drop in baseline; Figure 1I) because the boundaries of their corresponding point images do not overlap (Figure $1 \mathrm{~F}$ ). The behavioral results were in line with these predictions: The local abrupt-onset was associated with shorter SRTs, and the remote abrupt-onset was associated 
with longer SRTs (Figure 2). However, we found that the local abrupt-onset was associated with a momentary decrease in goal-directed activation (i.e., a drop in baseline; Figures 3 and 5). Although this decrease was indicative of spatial competition, it was immediately followed by a rebound in activation that resulted in a faster rise-to-threshold, and it was this rebound that produced the shorter SRTs associated with this condition (Figures 5 and 6).

\section{Underlying Mechanism}

Under the assumption of distance-dependent excitation/ inhibition between point images in the SCi (Marino, Trappenberg, et al., 2012; Trappenberg et al., 2001), the results suggest that point images associated with the goal and local abrupt-onset did not overlap and were mutually inhibitory. One possibility is that point images in the SCi are not fixed but may be shaped (sharpened) by topdown inputs in response to task demands (Schall, Sato, Thompson, Vaughn, \& Juan, 2004). For example, the goal-related point image may become narrower such that neighboring distractors fall outside the excitatory boundary in the inhibitory region and are therefore suppressed. However, such inhibition should delay, not facilitate saccade initiation. Moreover, the suppression was observed at most locations that contained the common feature defining distractors/abrupt-onsets (i.e., the color red; Figures 3C and 6) but not at other locations (namely, the target location in the control and distal abrupt-onset conditions; Figures 3B and 5A). This is indicative of a mechanism that enabled monkeys to quickly discount stimuli that shared the common nontarget feature, most likely acquired through extensive training with fixed stimulus colors. This is reminiscent of the type of experience-induced feature suppression of FEF neurons described by Bichot et al. (1996). However, although this may account for the suppression, it does not account for the subsequent rebound. Furthermore, the suppression and rebound were not entirely independent because suppression magnitude was correlated with accumulation rate (Figure 8). In other words, the momentary decrease in goal-directed activation associated with the local abrupt-onset appeared to subsequently boost neuronal excitability, acting to drive activation toward saccade threshold. This pattern is reminiscent of the action of certain types of neuronal ion channels, for example, T-type calcium channels (Cain \& Snutch, 2010; Huguenard, 1996). These channels will inactivate during steady depolarization (Isope, Hildebrand, \& Snutch, 2010), which in our paradigm would occur during presentation of the array (Figure 3A), which produces a persistent goal signal (25\% probability) before the target is revealed. Presentation of the local abrupt-onset then momentarily inhibits the neuron (release of GABA), producing the suppression response (transient reduction in discharge frequency, marked by downward arrow in Figure 3B). This inhibition would lead to a deinactivation of the T-type calcium channels (Williams, Toth, Turner, Hughes, \& Crunelli, 1997) so that the neurons can then respond more aggressively to the subsequent incoming excitatory goal signal (post inhibitory rebound, marked by upward arrow in Figure 3B). This mechanism could produce an accelerated accumulation rate and subsequently shorter SRTs. Such a mechanism relies upon non-linear cellular processes. Incorporation of such dynamics may be important for future biologically plausible models if a direct relationship with oculomotor behavior is established.

\section{Relation to Human Studies}

Although the oculomotor-capture task was designed to study the effect of salient stimuli on saccade programming, it is an extension of the attention-capture task (Theeuwes et al., 1999). Human studies have examined neural correlates of attention-capture during target selection using fMRI and ERPs. The capture of visual attention by irrelevant singletons is associated with enhanced activation over parietal and frontal regions (de Fockert \& Theeuwes, 2012; Talsma, Coe, Munoz, \& Theeuwes, 2010; de Fockert et al., 2004) and visual cortex (Mulckhuyse, Belopolsky, Heslenfeld, Talsma, \& Theeuwes, 2011). It is also associated with an enhancement of the N2pc ERP component (Hickey, McDonald, \& Theeuwes, 2006), which is related to the deployment of spatial attention. The general consensus from these studies is that visual attention is automatically captured by the salient distractor and is then voluntarily shifted toward the goal, which accounts for the delayed response. These studies support the dominant view of a frontal-parietal network in the control of visual attention. However, a growing number of influential studies also implicate the evolutionarily older SC as a crucial substrate in this regard (Zenon \& Krauzlis, 2012; Lovejoy \& Krauzlis, 2010; Ignashchenkova et al., 2004; McPeek \& Keller, 2004; Basso \& Wurtz, 1997; Kustov \& Robinson, 1996). Reasoning by analogy, if the salient taskirrelevant items in our study "captured" visual attention, it was certainly most associated with the local abrupt-onset (Figures 3 and 5). This would be consistent with the idea that attention was exogenously shifted away from the goal toward the local abrupt-onset. However, because of the subsequent rebound, this was associated with shorter, not longer, SRTs. To our knowledge, such nonlinear dynamics are not described by existing theories/ models of overt or covert selection. Whether this pattern depends on the type of training and experience described earlier remains to be determined. Highly trained animals with months of reward feedback could shape the visual attention system in a manner that is not typically seen in human studies (Awh, Belopolsky, \& Theeuwes, 2012). Future research would benefit from examination of neural correlates of human behavior after extensive training.

\section{Conclusion}

This study reported a simple behavior that was associated with a rather complex pattern of neuronal activation not readily explained by current models of the saccade system. 
The results revealed a pattern of excitation/inhibition across the SC visuomotor map that acted to facilitate optimal behavior in a typically difficult oculomotor task-a short duration suppression minimized the probability of capture by salient distractors, while a subsequent rebound boosted accumulation rate that ensured a fast goal-directed response. Such nonlinear dynamics in presaccadic activation should be incorporated into future biologically plausible models.

\section{Acknowledgments}

The authors thank Ann Lablans, Donald Brien, Sean Hickman, and Mike Lewis for outstanding technical assistance. This project was funded by the Human Frontiers Science Program, grant RGP0039-2005-C, the National Science Foundation (CRCNS grant BCS-0827764), and the Canadian Institutes of Health Research grant CNS-90910. D. P. M. was supported by the Canada Research Chair Program.

Reprint requests should be sent to Brian J. White, Centre for Neuroscience Studies, Queen's University, Botterell Hall, Rm 245, 18 Stuart Street, Kingston, Ontario, Canada, K7L 3N6, or via e-mail: brian.white@queensu.ca.

\section{Note}

1. Many SC neurons showed a burst of activation $\sim 40 \mathrm{msec}$ after saccade onset, which can be seen in the population average (Figure $5 \mathrm{H}$ ). This has been described as a postsaccadic visual response (Marino, Levy, et al., 2012; Li \& Basso, 2008), due to the RF rapidly moving from a stimulus as the eyes are launched to a new location.

\section{REFERENCES}

Awh, E., Belopolsky, A. V., \& Theeuwes, J. (2012). Top-down versus bottom-up attentional control: A failed theoretical dichotomy. Trends in Cognitive Science, 16, 437-443.

Basso, M. A., \& Wurtz, R. H. (1997). Modulation of neuronal activity by target uncertainty. Nature, 389, 66-69.

Behan, M., \& Kime, N. M. (1996). Intrinsic circuitry in the deep layers of the cat superior colliculus. Visual Neuroscience, 13, 1031-1042.

Bichot, N. P., Schall, J. D., \& Thompson, K. G. (1996). Visual feature selectivity in frontal eye fields induced by experience in mature macaques. Nature, 381, 697-699.

Bompas, A., \& Sumner, P. (2011). Saccadic inhibition reveals the timing of automatic and voluntary signals in the human brain. Journal of Neuroscience, 31, 12501-12512.

Born, S., \& Kerzel, D. (2011). Time-course of feature-based top-down control in saccadic distractor effects. Journal of Experimental Psychology: Human Perception and Performance, 37, 1689-1699.

Cain, S. M., \& Snutch, T. P. (2010). Contributions of T-type calcium channel isoforms to neuronal firing. Channels (Austin, Tex.), 4, 475-482.

Carpenter, R. H. S. (1988). Movements of the eyes. London: Pion.

de Fockert, J., Rees, G., Frith, C., \& Lavie, N. (2004). Neural correlates of attentional capture in visual search. Journal of Cognitive Neuroscience, 16, 751-759.

de Fockert, J. W., \& Theeuwes, J. (2012). Role of frontal cortex in attentional capture by singleton distractors. Brain $\mathcal{E}$ Cognition, 80, 367-373.
Dorris, M. C., \& Munoz, D. P. (1998). Saccadic probability influences motor preparation signals and time to saccadic initiation. Journal of Neuroscience, 18, 7015-7026.

Dorris, M. C., Olivier, E., \& Munoz, D. P. (2007). Competitive integration of visual and preparatory signals in the superior colliculus during saccadic programming. Journal of Neuroscience, 27, 5053-5062.

Fecteau, J. H., \& Munoz, D. P. (2005). Correlates of capture of attention and inhibition of return across stages of visual processing. Journal of Cognitive Neuroscience, 17, 1714-1727.

Glimcher, P. W., \& Sparks, D. L. (1992). Movement selection in advance of action in the superior colliculus. Nature, 355, 542-545.

Godijn, R., \& Theeuwes, J. (2002). Programming of endogenous and exogenous saccades: Evidence for a competitive integration model. Journal of Experimental Psychology: Human Perception and Performance, 28, 1039-1054.

Hanes, D. P., \& Schall, J. D. (1996). Neural control of voluntary movement initiation. Science, 274, 427-430.

Hays, A. V., Richmond, B. J., \& Optican, L. M. (1982). A UNIXbased multiple-process system for real-time data acquisition and control. WESCON Conference Proceedings, 1-10.

Hickey, C., McDonald, J. J., \& Theeuwes, J. (2006). Electrophysiological evidence of the capture of visual attention. Journal of Cognitive Neuroscience, 18, 604-613.

Huguenard, J. R. (1996). Low-threshold calcium currents in central nervous system neurons. Annual Review of Physiology, 58, 329-348.

Ignashchenkova, A., Dicke, P. W., Haarmeier, T., \& Thier, P. (2004). Neuron-specific contribution of the superior colliculus to overt and covert shifts of attention. Nature Neuroscience, 7, 56-64.

Isa, K., Phongphanphanee, P., Marino, R., Kaneda, K., Yanagawa, Y., Munoz, D. P., et al. (2009). The lateral interaction in the intermediate layers of the mouse superior colliculus slice. Neuroscience Research, 65, S172.

Isope, P., Hildebrand, M. E., \& Snutch, T. P. (2010). Contributions of T-type voltage-gated calcium channels to postsynaptic calcium signaling within purkinje neurons. Cerebellum, 11, 651-665.

Keller, E. L., \& McPeek, R. M. (2002). Neural discharge in the superior colliculus during target search paradigms. Annals of the New York Academy of Sciences, 956, 130-142.

Kim, B., \& Basso, M. A. (2008). Saccade target selection in the superior colliculus: A signal detection theory approach. Journal of Neuroscience, 28, 2991-3007.

Krauzlis, R. J., Liston, D., \& Carello, C. D. (2004). Target selection and the superior colliculus: Goals, choices and hypotheses. Vision Research, 44, 1445-1451.

Kustov, A. A., \& Robinson, D. L. (1996). Shared neural control of attentional shifts and eye movements. Nature, 384, 74-77.

Leonard, C. J., \& Luck, S. J. (2011). The role of magnocellular signals in oculomotor attentional capture. Journal of Vision, $11,1-12$.

Li, X., \& Basso, M. A. (2008). Preparing to move increases the sensitivity of superior colliculus neurons. Journal of Neuroscience, 28, 4561-4577.

Lovejoy, L. P., \& Krauzlis, R. J. (2010). Inactivation of primate superior colliculus impairs covert selection of signals for perceptual judgments. Nature Neuroscience, 13, 261-266.

Ludwig, C. J., Ranson, A., \& Gilchrist, I. D. (2008). Oculomotor capture by transient events: A comparison of abrupt onsets, offsets, motion, and flicker. Journal of Vision, 8, 1-16.

Marino, R. A., Levy, R., Boehnke, S., White, B. J., Itti, L., \& Munoz, D. P. (2012). Linking visual response properties in the superior colliculus to saccade behavior. The European Journal of Neuroscience, 35, 1738-1752. 
Marino, R. A., Rodgers, C. K., Levy, R., \& Munoz, D. P. (2008). Spatial relationships of visuomotor transformations in the superior colliculus map. Journal of Neurophysiology, 100, 2564-2576.

Marino, R. A., Trappenberg, T. P., Dorris, M., \& Munoz, D. P. (2012). Spatial interactions in the superior colliculus predict saccade behavior in a neural field model. Journal of Cognitive Neuroscience, 24, 315-336.

Mcllwain, J. T. (1986). Point images in the visual system: New interest in an old idea. Trends in Neurosciences, 9, 354-358.

McPeek, R. M., \& Keller, E. L. (2002). Saccade target selection in the superior colliculus during a visual search task. Journal of Neurophysiology, 88, 2019-2034.

McPeek, R. M., \& Keller, E. L. (2004). Deficits in saccade target selection after inactivation of superior colliculus. Nature Neuroscience, 7, 757-763.

McSorley, E., McCloy, R., \& Lyne, C. (2012). The spatial impact of visual distractors on saccade latency. Vision Research, 60, $61-72$.

Meeter, M., Van der Stigchel, S., \& Theeuwes, J. (2010). A competitive integration model of exogenous and endogenous eye movements. Biological Cybernetics, 102, 271-291.

Meredith, M. A., \& Ramoa, A. S. (1998). Intrinsic circuitry of the superior colliculus: Pharmacophysiological identification of horizontally oriented inhibitory interneurons. Journal of Neurophysiology, 79, 1597-1602.

Miyashita, N., \& Hikosaka, O. (1996). Minimal synaptic delay in the saccadic output pathway of the superior colliculus studied in awake monkey. Experimental Brain Research, 112, 187-196.

Mulckhuyse, M., Belopolsky, A. V., Heslenfeld, D., Talsma, D., \& Theeuwes, J. (2011). Distribution of attention modulates salience signals in early visual cortex. PloS One, 6, e20379.

Munoz, D. P., \& Istvan, P. J. (1998). Lateral inhibitory interactions in the intermediate layers of the monkey superior colliculus. Journal of Neurophysiology, 79, 1193-1209.

Munoz, D. P., Waitzman, D. M., \& Wurtz, R. H. (1996). Activity of neurons in monkey superior colliculus during interrupted saccades. Journal of Neurophysiology, 75, 2562-2580.

Munoz, D. P., \& Wurtz, R. H. (1995). Saccade-related activity in monkey superior colliculus. I. Characteristics of burst and buildup cells. Journal of Neurophysiology, 73, 2313-2333.

Paré, M., \& Hanes, D. P. (2003). Controlled movement processing: Superior colliculus activity associated with countermanded saccades. Journal of Neuroscience, 23, 6480-6489.

Port, N. L., \& Wurtz, R. H. (2009). Target selection and saccade generation in monkey superior colliculus. Experimental Brain Research, 192, 465-477.

Purcell, B. A., Schall, J. D., Logan, G. D., \& Palmeri, T. J. (2012). From salience to saccades: Multiple-alternative gated stochastic accumulator model of visual search. Journal of Neuroscience, 32, 3433-3446.

Robinson, D. A. (1963). A method of measuring eye movement using a scleral search coil in a magnetic field. IEEE Transactions on Biomedical Engineering, 10, 137-145.

Rodgers, C. K., Munoz, D. P., Scott, S. H., \& Paré, M. (2006). Discharge properties of monkey tectoreticular neurons. Journal of Neurophysiology, 95, 3502-3511.
Schall, J. D., Sato, T. R., Thompson, K. G., Vaughn, A. A., \& Juan, C. H. (2004). Effects of search efficiency on surround suppression during visual selection in frontal eye field. Journal of Neurophysiology, 91, 2765-2769.

Schall, J. D., \& Thompson, K. G. (1999). Neural selection and control of visually guided eye movements. Annual Review of Neuroscience, 22, 241-259.

Shen, K., \& Paré, M. (2007). Neuronal activity in superior colliculus signals both stimulus identity and saccade goals during visual conjunction search. Journal of Vision, 7, 1-13.

Sparks, D. L. (2002). The brainstem control of saccadic eye movements. Nature Reviews Neuroscience, 3, 952-964.

Talsma, D., Coe, B., Munoz, D. P., \& Theeuwes, J. (2010). Brain structures involved in visual search in the presence and absence of color singletons. Journal of Cognitive Neuroscience, 22, 761-774.

Theeuwes, J., De Vries, G. J., \& Godijn, R. (2003). Attentional and oculomotor capture with static singletons. Perception $\mathcal{E}$ Psychophysics, 65, 735-746.

Theeuwes, J., Kramer, A. F., Hahn, S., Irwin, D. E., \& Zelinsky, G. J. (1999). Influence of attentional capture on oculomotor control. Journal of Experimental Psychology: Human Perception and Performance, 25, 1595-1608.

Thompson, K. G., Hanes, D. P., Bichot, N. P., \& Schall, J. D. (1996). Perceptual and motor processing stages identified in the activity of macaque frontal eye field neurons during visual search. Journal of Neurophysiology, 76, 4040-4055.

Trappenberg, T. P., Dorris, M. C., Munoz, D. P., \& Klein, R. M. (2001). A model of saccade initiation based on the competitive integration of exogenous and endogenous signals in the superior colliculus. Journal of Cognitive Neuroscience, 13, 256-271.

Walker, R., Deubel, H., Schneider, W. X., \& Findlay, J. M. (1997). Effect of remote distractors on saccade programming: Evidence for an extended fixation zone. Journal of Neurophysiology, 78, 1108-1119.

White, B. J., Boehnke, S. E., Marino, R. A., Itti, L., \& Munoz, D. P. (2009). Color-related signals in the primate superior colliculus. Journal of Neuroscience, 29, 12159-12166.

White, B. J., Gegenfurtner, K. R., \& Kerzel, D. (2005). Effects of structured nontarget stimuli on saccadic latency. Journal of Neurophysiology, 93, 3214-3223.

White, B. J., \& Munoz, D. P. (2011a). Separate visual signals for saccade initiation during target selection in the primate superior colliculus. Journal of Neuroscience, 31, 1570-1578.

White, B. J., \& Munoz, D. P. (2011b). The superior colliculus. In S. Liversedge, I. Gilchrist, \& S. Everling (Eds.), Oxford handbook of eye movements (1st ed., pp. 195-213). Oxford: Oxford University Press.

White, B. J., Theeuwes, J., \& Munoz, D. P. (2012). Interaction between visual- and goal-related neuronal signals on the trajectories of saccadic eye movements. Journal of Cognitive Neuroscience, 24, 707-717.

Williams, S. R., Toth, T. I., Turner, J. P., Hughes, S. W., \& Crunelli, V. (1997). The "window" component of the low threshold $\mathrm{Ca} 2+$ current produces input signal amplification and bistability in cat and rat thalamocortical neurones. The Journal of Physiology, 505, 689-705.

Zenon, A., \& Krauzlis, R. J. (2012). Attention deficits without cortical neuronal deficits. Nature, 489, 434-437. 